\title{
Estudio descriptivo sobre el bienestar subjetivo psicológico en una muestra de habitantes de la Patagonia austral
}

Descriptive study about psychological subjective well-being in southern Patagonian habitants

\author{
Enrique Puebla Rosalessa, Sergio Avendaño Alarcón ${ }^{a} \&$ Claudia Estrada Goic ${ }^{a}$ \\ aEscuela de Psicología, Universidad de Magallanes. \\ $>$ claudia.estrada@umag.cl
}

\begin{abstract}
RESUMEN
El objetivo central de la presente investigación fue caracterizar el bienestar psicológico subjetivo de una parte de la población de la Patagonia Austral. Un grupo de 400 personas constituyeron la muestra no aleatoria autoselectiva participaron en este estudio. El diseño no experimental descriptivo utilizó un cuestionario autoaplicado para medir el bienestar psicológico. Los resultados indican que la población muestra un promedio general alto de bienestar observándose diferencias en algunos dominios y dimensiones de esta variable, particularmente para el nivel socioeconómico, el estado civil y la escolaridad. Contrario a lo señalado por la literatura no se observaron diferencias significativas según género o edad. Los resultados se discuten en torno a las diferencias observadas en comparación con estudios de otras realidades culturales y su potencial interés para la salud mental de los individuos sometidos a las condiciones ambientales características de la Patagonia.
\end{abstract}

PALABRAS CLAVE: Calidad de Vida, Bienestar Psicológico Subjetivo, Patagonia Austral.

\begin{abstract}
The primary objective of the present research was to characterize the subjective wellbeing of the southern Patagonia population. A group of 400 people constituted the no random auto selective sample part in this study. The non-experimental cross design used a selfapply questionary to measure the psychological, subjective well-being. The results indicate that the population shows a high overall average of well-being, with differences observed in some domains and dimensions of this variable, particularly for socioeconomic level, marital


status, and schooling. Contrary to what was stated in the literature, no significant differences were observed according to gender or age. The results are discussed around the observed differences between studies with other cultures realities and its potential interest for the mental health of the individuals, subjected to environmental conditions characteristic of Patagonia.

KEY WORDS: Quality Life, Subjective Well-Being, Austral Patagonia.

\section{INTRODUCCIÓN}

En las últimas décadas ha aumentado el interés por comprender y mejorar la calidad de vida de las personas. Uno de los aspectos abordados son los factores que inciden sobre el bienestar y la forma en que se puede incrementar lo que ha conducido a una serie de investigaciones (Arita, 2005; Argyle, 1993; Casullo, 2002; Contini, Cohen, Figueroa \& Coronel, 2006; Cuadra \& Florenzano, 2003; Dolan, Peasgood \& White, 2008; Keyes, Shmotkin \& Ryff, 2002; Moyano \& Ramos, 2007; Suh, Diener \& Fujita 1996; etc.). Este énfasis ocurre en forma paralela al nacimiento de la llamada Psicología Positiva, que se interesa por investigar acerca de la felicidad, el optimismo, la sabiduría y otros temas relacionados con las fortalezas y virtudes humanas. (Carr, 2007; Cuadra \& Florenzano, 2003). A esto se suma la creciente preocupación por contar con más eficientes indicadores de calidad de vida para mejorar las políticas de cambio social (Keyes, Shmotkin \& Ryff, 2002).

El concepto de calidad de vida nace alrededor de los años 50 con relación al debate sobre el deterioro de las condiciones de vida urbana y del medio ambiente. Inicialmente se consideraron medidas puramente objetivas, como las económicas y las sociales (Gómez \& Sabeh, 2001). Consecuentemente, el indicador universal para medir la calidad de vida fue el Índice de Desarrollo Humano (IDH), establecido por el Programa de las Naciones Unidas para el Desarrollo (PNUD) con el propósito de cuantificar el grado de desarrollo de los países. Este indicador que se calcula considerando niveles de ingreso, educación y esperanza de vida de la población (PNUD, 1990; Arita, 2005). Fue en un segundo momento, y a partir de las diferencias observadas entre estas medidas objetivas y las discordancias con la percepción de la población, que se comenzó a considerar la importancia de los aspectos subjetivos de la calidad de vida. En las décadas del 70 y 80 se diferenciaron los indicadores sociales de los de calidad de vida tradicionales, comenzando a entenderse éste último como un concepto integral (Gómez y Sabeh, 2001). Aunque aun no existe un consenso respecto a este constructo, una proposición reciente sostiene que la calidad de vida implicaría una combinación de elementos objetivos y subjetivos. Incluiría tanto el nivel de satisfacción que experimenta un individuo respecto de sus condiciones vitales, las que se componen de elementos objetivos (condiciones de vida), y subjetivos (satisfacción personal) (Borthwick-Duffy, 1992; Ardila, 2003). Posteriormente fue definida por la OMS como "la percepción que un individuo tiene de 
su lugar en la existencia, en el contexto de la cultura y del sistema de valores en los que vive y en relación con sus objetivos, sus expectativas, sus normas, sus inquietudes Se trata de un concepto muy amplio que está influido de modo complejo por la salud física del sujeto, su estado psicológico, su nivel de independencia, sus relaciones sociales, así como su relación con los elementos esenciales de su entorno" (The WHOQOL Group, 1995, p. 1405). De esta manera la percepción que cada ser humano tiene respecto de sus condiciones de vida lo que va a comenzar a ser investigado, aspecto que ha sido denominado bienestar psicológico subjetivo (Sánchez-Canovas, 1998), simplemente bienestar subjetivo (Arita, 2005), o felicidad sentida (Veenhoven, 2000 en Arita, 2005).

Durante más de cien años la observación del comportamiento fue la estrategia de evaluación del bienestar. Luego de numerosos intentos de mejorar su medición, el auto-reporte se reveló como la medida más confiable para esta variable. (Dolan et al. 2008). Un metanálisis realizado en el 2001 (Ryan \& Deci, 2001) clasifica las investigaciones sobre bienestar en dos grandes tradiciones: una relacionada con la felicidad (bienestar hedónico), y otra asociada al desarrollo del potencial humano (bienestar eudaemónico). Tradiciones que actualmente están relacionadas al constructo bienestar subjetivo (tradición hedónica) y con el constructo bienestar psicológico (tradición eudaemónica) (ver también Keyes, et al 2002). Mientras tradición del bienestar subjetivo se preocupó por el estudio de los afectos y la satisfacción con la vida, la tradición del bienestar psicológico centró su atención en el desarrollo de las capacidades y el crecimiento personal. Ryff (1989, en Díaz et al. 2006) propone un modelo multidimensional del bienestar psicológico constituido por cinco dimensiones: aceptación de si mismo, vínculos psicosociales, autonomía, control de situaciones y proyectos. A este modelo otros autores han incluido la dimensión felicidad (Moyano \& Ramos, 2007).

La dimensión de aceptación de sí mismo, es un criterio central del bienestar, que se caracteriza por una apreciación positiva de sí mismo y aceptación de todos los aspectos que caracterizan al sujeto tanto positivos como negativos (Díaz et al. 2006). Una persona con una alta aceptación de sí mismo muestra conformidad con su cuerpo, con su forma de ser, con su pasado y acepta sus equivocaciones, sintiéndose impulsado a mejorar (Castro, 2002). El control de las situaciones se refiere a la capacidad de poder manejar de forma efectiva los factores del medio ambiente y la propia vida, de modo de influir en el entorno y generar los cambios necesarios para satisfacer las necesidades y motivaciones propias. (Díaz et al. 2006; Castro, 2002). La dimensión de vínculos psicosociales se relaciona con la capacidad para establecer relaciones de confianza con los demás, y se asocia a la capacidad de amar variable que constituye un importante factor de bienestar y salud mental (Díaz et al. 2006). Con relación a la dimensión de autonomía, esta se refiere a la capacidad que tiene las personas para tomar sus propias decisiones y expresar su individualidad en los distintos contextos sociales, resistiendo la presión social y autorregulando la propia conducta. Respecto a la dimensión de proyectos esta es entendida como la capacidad de las personas para colocarse metas y proyectos en la vida, ya que éstas son las que otorgan de propósito y significado a 
sus vidas, dando estructura y sentido a la misma (Barrientos, 2006). La dimensión felicidad, por su parte, hace referencia a un estado psicológico que abarca un aspecto emocional y otro cognitivo, el cual se configura como un factor protector frente al surgimiento de fenómenos psicopatológicos (Moyano \& Ramos, 2007). Cabe precisar que estas dimensiones no se dan de forma aislada, sino que están fuertemente influenciadas por los contextos, microsocial y macrosocial, desde donde surge el concepto de dominios de satisfacción: el área laboral, el área de las relaciones interpersonales, el área material y el área subjetiva (Sanchez-Cánovas, 1998).

De este modo se puede definir al bienestar psicológico como el grado en el que una persona evalúa en términos favorables su vida, asociado principalmente a la sensación de control percibido sobre las situaciones, la aceptación de sí mismo, la satisfacción con los logros alcanzados, la capacidad de establecer vínculos psicosociales satisfactorios y sentido de autodeterminación o autonomía, todos los cuales se dan dentro de los dominios laboral, material, subjetivo y relaciones interpersonales.

Numerosas son las investigaciones actuales que intentan determinar cuales son los factores que inciden en el bienestar subjetivo, así por ejemplo se han estudiado la influencia que tendrían los eventos recientes (Suh et al. 1996), la remuneración (Diener \& Biswas 2002), la edad (Blanchflower \& Oswald, 2008), el género, la personalidad, la educación y el desempleo, entre otras tantas (Para una revisión exhaustiva ver Dolan et al. 2008). Con relación al nivel socio económico, la literatura señala que obtener mayores ingresos podría aumentar los niveles de bienestar subjetivo únicamente en los estratos económicos bajos (Diener \& Biswas, 2002; Ardila, 2003). Un ingreso adicional no incrementaría la sensación de bienestar en aquellas personas cuyo grupo de referencia obtienen un aumento similar (Dolan, Peasgood \& White, 2008). Incluso, se ha observado que poner énfasis en la adquisición de riquezas puede ser contraproducente para logro de la felicidad (Diener \& Biswas, 2002), y que la influencia de la situación económica sobre la sensación de bienestar subjetivo no depende tanto del ingreso, sino de las expectativas y del número de necesidades que determiner cual es el grado percibimos satisfechas (Garduño, Salinas \& Rojas, 2005). Respecto al estado civil, las investigaciones muestran que las personas casadas son las que tienen el más alto índice de bienestar subjetivo mientras que los separados el más bajo, incluso menor que los divorciados y viudos (Dolan et al. 2008). En términos de género, las mujeres separadas son las que alcanzan las más altas puntuaciones en las escalas de depresión, paranoidismo y esquizofrenia (Barrio \& Domenech, 1993), mientras que las solteras muestran mayor bienestar que las casadas (Wildman \& Jones, 2002). Existiría una relación positiva entre el nivel educacional y el nivel de Bienestar subjetivo que tendría mayor impacto en los países donde las personas con baja educación obtienen un menor ingreso. (Dolan et al. 2008). Aunque aun se requieren más investigaciones para establecer con mayor precisión las fuentes causales del bienestar subjetivo, dado el cúmulo de información existente se reconocen como variables relevantes la remuneración, salud, relaciones personales, situación laboral y estado 
civil (Dolan et al. 2008). Asimismo, la evidencia señala que los bajos índices de salud, la separación, el desempleo y la falta de contacto social están fuertemente asociados a un bajo nivel de Bienestar Subjetivo (Dolan et al. 2008).

En la dirección de lo hasta aquí explicitado, la presente investigación se ocupa de determinar cuál es el grado de satisfacción que posee una muestra de los habitantes de la Patagonia Austral en los dominios y dimensiones del bienestar, así como el bienestar subjetivo general. Mediante la exploración de los niveles de satisfacción y los posibles efectos de interacción de las variables sociodemográficas, se busca contribuir a generar un marco de conocimiento que permitiría la formulación de intervenciones, en factores claves del bienestar psicosocial, orientadas a mejorar los índices de salud mental de nuestra población.

\section{MÉTODO}

\section{Participantes}

La muestra no probabilística accidental estuvo compuesta por 400 habitantes de la Patagonia Austral (residentes en la ciudad de Punta Arenas), con edades comprendidas entre los 15 a los 90 años. De ellos un 50,8\% corresponden al género femenino y un 49,3\% al masculino. Asimismo, un 35,3\% corresponde a personas entre 26 y 44 años; un 25\% entre los 17 a 25 años, un 22,5\% entre 45 y 64 años, y un 17,3\% entre 65 a 90 años. Un 29,3\% tiene Estudios Universitarios Incompletos, un 20,3\% Estudios Medios Completos, un 17,8\% Estudios Técnicos Completos, un 10,8\% Estudios Universitarios Completos, un 8,8\% Estudios Medios Incompletos, un 5,8\% Estudios Técnicos Incompletos, un 4,5\% Estudios Básicos, y un 3\% Estudios de Post-Grado. Respecto al estado civil un 41\% son solteros; un 37,5\% casados; un $8,5 \%$ se encontraban conviviendo; un $5,5 \%$ separado; un $5,3 \%$ viudo; y un 2,3\% divorciado legalmente. Por último, con relación al nivel socio económico se observó que un 59,5\% de la población se clasifica en el Nivel Socio Económico Medio; un 20,5\% en el Nivel Socio Económico Medio Bajo; un 13,5\% en el Nivel Socio Económico Medio Alto; un 5,5\% en el Nivel Socio Económico Bajo; y un 1\% en el Nivel Socio Económico Alto.

\section{Diseño}

Se utilizó un diseño no experimental transversal descriptivo.

\section{Instrumentos}

Cuestionario de bienestar Psicológico: Cuestionario creado por los autores de la presente investigación que mide el nivel de bienestar psicológico que las personas experimentan respecto de sus vidas. Fue construido integrando dos modelos distintos de bienestar; dominios 
de bienestar: subjetivo, laboral, relaciones interpersonales y material; y dimensiones de bienestar: control de situaciones, aceptación de sí mismo, vínculos psicosociales, proyectos, autonomía y felicidad. El instrumento está compuesto por 44 ítems, en el que cada afirmación mide un dominio y una dimensión simultáneamente que se responden en base a una escala Likert de 5 puntos que va desde el 1: “Totalmente en desacuerdo" hasta el 5: "Totalmente de acuerdo”. (Alfa de Cronbach: 0.95). El instrumento posee validez de constructo (evaluación de jueces expertos) y en una investigación anterior se estableció su validez concurrente. Su confiabilidad actual fue de Alfa .93 (Cronbach).

\section{Procedimiento}

Cada participante fue contactado en su lugar de estudio, trabajo o habitación. De forma voluntaria aceptaron participar en la investigación, firmaron un consentimiento informado asegurando la confidencialidad de los datos y se auto aplicaron los instrumentos. Al finalizar se expusieron los objetivos centrales del estudio y se les indico la forma de acceder a sus resultados individuales.

\section{RESULTADOS}

\section{Descriptivos}

Al analizar los resultados descriptivos por dimensiones del bienestar psicológico, esto es, (1) control de situaciones, (2) aceptación de sí mismo, (3) vínculos psicosociales, (4) autonomía, (5) proyectos y (6) felicidad, podemos observar que todas ellas muestran promedios dentro de un alto nivel de bienestar psicológico. La dimensión que mejor evalúan los participantes es la de Proyectos, seguida por la de Control de Situaciones y la de Aceptación de sí mismo (Ver tabla 1).

Los resultados por dominios del bienestar psicológico, esto es, (1) área Material, (2) Relaciones Interpersonales (3) Subjetiva, (4) Laboral, indican que todos ellos se encuentran dentro de un nivel alto de bienestar psicológico. El dominio mejor evaluado fue el de Relaciones Interpersonales, seguido por el Laboral (Ver tabla 2).

$\mathrm{Al}$ analizar el bienestar psicológico total de la muestra se observa que el nivel promedio de bienestar que presentan los participantes es alto $(M=4,15 ; D E=0,46)$, distribuyéndose de la siguiente forma: Alto Bienestar 87,3\%; Bienestar Intermedio 12,3\%; y Bajo Bienestar $0,5 \%$.

Los resultados según dimensiones señalan que, en Felicidad, se observan efectos principales únicamente para estado civil y NSE. En el caso de estado civil, los participantes casados $(M=4,20, D E=0,75)$ muestran mayor bienestar que los que se encuentran conviviendo $(M=4,16, D E=0,62)$, los solteros $(M=4,07, D E=0,88)$, los separados o divorciados $(M=3,95, D E=$ 
ESTUDIO SOBRE BIENESTAR SUBJETIVO PSICOLÓGICO

Tabla 1. Media, Desviación Estándar y Distribución de Porcentajes de las dimensiones del bienestar psicológico.

\begin{tabular}{|c|c|c|c|c|c|}
\hline \multirow[b]{3}{*}{ Control Situaciones } & \multirow[b]{2}{*}{$M$} & \multirow[b]{2}{*}{ D.E. } & \multicolumn{3}{|c|}{ Nivel de Bienestar Psicológico } \\
\hline & & & Bajo & Medio & Alto \\
\hline & 4,21 & 0,52 & 0,5 & 13,3 & 86,3 \\
\hline Aceptación de sí mismo & 4,12 & 0,54 & 0,3 & 17,3 & 82,5 \\
\hline Vínculos Psicosociales & 4,11 & 0,56 & 0,8 & 20,3 & 79,0 \\
\hline Autonomía & 4,09 & 0,61 & 0,8 & 18,5 & 80,8 \\
\hline Proyectos & 4,31 & 0,50 & 0,5 & 8,0 & 91,5 \\
\hline Felicidad & 4,09 & 0,56 & 1,3 & 18,0 & 80,8 \\
\hline
\end{tabular}

Fuente: Elaboración propia.

Tabla 2. Media, Desviación Estándar y Distribución de P orcentajes de los dominios del bienestar psicológico.

\begin{tabular}{|c|c|c|c|c|c|}
\hline & & & \multicolumn{3}{|c|}{ Nivel de Bienestar Psicológico } \\
\hline & $M$ & D.E. & Bajo & Medio & Alto \\
\hline Material & 4,12 & 0,54 & 0,5 & 13,3 & 86,3 \\
\hline Subjetivo & 4,11 & 0,51 & 0,3 & 17,3 & 82,5 \\
\hline Laboral & 4,13 & 0,53 & 0,8 & 20,3 & 79,0 \\
\hline Relaciones Interpersonales & 4,27 & 0,51 & 1,3 & 18,0 & 80,8 \\
\hline
\end{tabular}

Fuente: Elaboración propia.

$0,57)$ y los viudos $(M=3,89, D E=0,60), F(4,395)=2,95, p<, 02$. En relación a NSE, se observa que los participantes de un rango alto o muy alto $(M=4,27, D E=0,68)$ muestran mayor bienestar que las de otros grupos, $F(2,397)=6,08, p<, 01$. También existe un efecto de interacción entre las variables NSE y género $(F(2,397)=2,92, p<0,05)$. Las diferencias más importantes se sitúan en el género masculino, en donde los hombres del NSE alto $(M=4,35, D E=0,70)$ presentan un promedio de bienestar significativamente mayor que los otros grupos.

En la dimensión Autonomía, se observa únicamente un efecto de interacción entre las variables NSE y edad $(F(6,397)=2,40, p<, 03)$, no observándose efectos principales significativos. Las diferencias más importantes se dan en el NSE muy alto o alto, en donde las personas entre 45 a 64 años $(M=4,37, D E=0,68)$ muestran un mayor nivel de bienestar en la dimensión autonomía que los otros grupos de edad y NSE.

En la dimensión Proyectos, se observa un efecto principal únicamente para estado civil. Los participantes casados $(M=4,40, D E=0,64)$ muestran mayor bienestar que los otros grupos $F(4,395)=2,87, p<, 02$.

En la dimensión Control de Situaciones, se observan efectos principales para las variables estado civil y NSE. En el caso de estado civil, los participantes casados ( $M=4,26$, 
$D E=0,69)$ muestran el mayor promedio de bienestar $F(4,395)=2,89, p<, 02$. Con relación a NSE, se observa que las personas de un rango alto o muy alto $(M=4,28, D E=0,62)$ son las más favorecidas en bienestar, $F(1,398)=4,89, p<, 03$. Existe un efecto de interacción entre las variables estado civil y $\operatorname{NSE}(F(8,391)=2,18, p<0,02)$. Las diferencias más importantes se sitúan en las personas separadas, en donde las de un NSE alto $(M=4,69, D E=0,52)$ presentan un promedio de bienestar significativamente mayor que las de un NSE medio $(M=4,32, D E=$ $0,53)$ y bajo $(M=3,39, D E=0,51)$.

En la dimensión Vínculos Psicosociales, se observa un efecto principal únicamente para la variable estado civil, en donde los participantes solteros $(M=4,23, D E=0,90)$ muestran mayor bienestar que los otros grupos, $F(4,395)=5,32, p<, 001$. Existe un efecto de interacción entre NSE y escolaridad $(F(5,394)=2,91, p<, 01)$. Las diferencias más importantes se sitúan en la escolaridad básica, donde las personas con un NSE alto $(M=4,21, D E=0,55)$ presentan un promedio de bienestar significativamente mayor que las de un NSE medio $(M=4,11, D E=$ $0,57)$ y bajo $(M=3,91, D E=0,59)$. Asimismo se observa un efecto de interacción entre las variables estado civil y escolaridad $(F(8,391)=1,97, p<, 05)$. Las diferencias se sitúan en la escolaridad básica, donde los individuos que se encuentran conviviendo $(M=4,56, D E=0,55)$ presentan un promedio de bienestar significativamente mayor a los otros grupos.

En la dimensión Aceptación de si mismo, se observan efectos principales únicamente para las variables estado civil y NSE. En el caso de estado civil, los participantes casados $(M=$ $4,24, D E=0,70)$ muestran mayor bienestar que los otros grupos $F(4,395)=3,94, p<, 06$. En relación a NSE se observa que los participantes de un rango alto o muy alto $(M=4,27, D E=$ $0,64)$ muestran mayor bienestar, $F(2,397)=5,16, p<, 01$. Existe un efecto de interacción entre las variables género y NSE $(F(2,397)=2,88, p<0,058)$. En el género masculino los hombres con un NSE alto $(M=4,36, D E=0,66)$ presentan un promedio de bienestar significativamente mayor que los otros. Asimismo se observa un efecto de interacción entre las variables estado civil y escolaridad $(F(8,391)=1,89, p<0,062)$. En la escolaridad básica las personas que se encuentran conviviendo $(M=4,50, D E=0,50)$ presentan un promedio de bienestar significativamente mayor que los otros.

En resumen, en el caso de las dimensiones del bienestar psicológico las variables sociodemográficas relevantes son el estado civil y el NSE. Son los casados y aquellos de NSE alto los que presentan mayor bienestar. Una excepción a este patrón se observa para la dimensión de vínculos sociales en la que los solteros salen favorecidos.

Al evaluar el dominio Laboral del bienestar psicológico se observan efectos principales únicamente para estado civil y nivel socio económico (NSE), no existiendo efectos de interacción significativos. En el caso del estado civil, los participantes casados $(M=4,20$, $D E=0,70)$ muestran mayor bienestar que todos los otros grupos, $F(4,395)=2,99, p<, 02)$. Respecto al nivel socio económico, los participantes en el rango alto o muy alto $(M=4,25, D E$ $=0,64)$ muestran mayor bienestar que los del rango medio $(M=4,21, D E=0,77)$ y de bajo o muy bajo $(M=3,93, D E=0,63), F(2,397)=3,79, p<, 02$. 
En el dominio Relaciones Interpersonales, se observa un efecto principal únicamente para estado civil, en donde los participantes solteros $(M=4,33, D E=0,81)$ muestran mayor bienestar que los que se encuentran conviviendo $(M=4,33, D E=0,56)$, los casados $(M=4,32$, $D E=0,67)$, los separados ( $M=4,13, D E=0,52)$ y los viudos $(M=4,04, D E=0,55), F(4,395)=4,41$, $p<, 002$. Existe un efecto de interacción entre las variables NSE y nivel de estudios $(F(5,394)=$ $2,15, p<0,06)$. Las diferencias más importantes se sitúan en el rango alto de ingreso, en donde las personas de escolaridad técnica $(M=4,63, D E=0,49)$ presentan un promedio de bienestar significativamente mayor que los otros niveles de estudio.

En el dominio Subjetivo, se observan efectos principales únicamente para estado civil y NSE. En el caso de estado civil, los participantes casados $(M=4,22, D E=0,66)$ muestran mayor bienestar que los participantes de otros estados civiles, $F(4,395)=4,34, p<, 002$. Respecto al NSE, se observa que los participantes del rango alto o muy alto $(M=4,24, D E=0,60)$ muestran mayor bienestar que el rango medio $(M=4,15, D E=0,73)$ y bajo o muy bajo $(M=$ $3,90, D E=0,60), F(2,397)=4,62, p<0,011$. Existe un efecto de interacción entre las variables género y NSE $(F 2,397)=3,17, p<, 044)$. Las diferencias más importantes se sitúan en el género masculino, donde los del NSE alto $(M=4,35, D E=0,62)$ presentan un promedio de bienestar significativamente mayor que los otros dos. Otra interacción significativa se da entre las variables NSE y escolaridad $(F(5,394)=2,31, p<0,045)$. La diferencia más importante se presenta en la escolaridad básica, en donde los del rango alto $(M=4,50, D E=0,48)$ muestran un promedio de bienestar significativamente mayor que el rango medio $(M=3,97, D E=0,50)$ y bajo $(M=3,77, D E=0,52)$. Asimismo se observa una interacción entre las variables Estado Civil y Escolaridad $(F(8,391)=1,92, p<0,057)$. La diferencia más significativa se presenta en la escolaridad básica, en donde los participantes que se encuentran conviviendo $(M=4,55$, $D E=0,48$ ) muestran un promedio de bienestar significativamente superior a los otros grupos.

En el dominio Material, se observan efectos principales para las variables escolaridad, estado civil y NSE, no existiendo efectos de interacción significativos. En el caso del estado civil, los participantes que se encuentran conviviendo $(M=4,21, D E=0,61)$ muestran mayor bienestar que los casados $(M=4,19, D E=0,75)$, solteros $(M=4,11, D E=0,88)$, viudos $(M=3,99, D E=0,60)$ y separados o divorciados $(M=3,91, D E=0,57), F(4,395)=3,71, p<0,006$. Respecto al NSE, se observa que los del rango alto o muy alto $(M=4,29, D E=0,68)$ muestran mayor bienestar que los del rango medio $(M=4,17, D E=0,82)$ y bajo o muy bajo $(M=3,94, D E=0,67), F(2,397)=4,89$, $p<0,008$. Y en relación a la escolaridad, se observa que los participantes técnicos $(M=4,24$, $D E=0,71)$ muestran mayor bienestar que los universitarios $(M=4,09, D E=0,85)$, con estudios medios $(M=4,07, D E=0,70)$ y básicos $(M=3,95, D E=0,57), F(3,396)=2,85, p<, 04$.

En resumen, al tratarse de dominios, los promedios mayores de bienestar se vinculan con la pertenencia a un nivel socioeconómico alto o muy alto, tal y como se observó para el caso de las dimensiones. El estado civil también se presenta como una variable relevante en la que los casados presentan mayor bienestar en lo laboral y subjetivo, mientras que los solteros los superan en las relaciones interpersonales y los convivientes en lo material. 
Por último, al evaluar el bienestar psicológico total según edad, género, NSE, escolaridad y estado civil, se observan efectos principales únicamente para estado civil y NSE. Los resultados reproducen lo observado anteriormente en los que los participantes casados y aquellos de NSE alto o muy alto.

\section{DISCUSIÓN Y CONCLUSIÓN}

El propósito de este estudio fue describir el bienestar subjetivo de los habitantes de la Patagonia Austral en una muestra de 400 personas, constatándose que el promedio de bienestar subjetivo general de la población es alto. Este resultado puede haberse originado en un defecto en la escala de medición del instrumento, (por cuanto este levanta información respecto al nivel de acuerdo con cada afirmación y no sobre la frecuencia de la vivencia asociada), al sesgo negativo conocido de la medición de esta variable incluso a nivel transcultural, puede deberse a que la muestra fue autoseleccionada, así como también el hecho de encontrarnos en una región señalada sistemáticamente como una de las mejores para vivir en Chile. Investigaciones en progreso intentan dilucidar cuál de estas explicaciones es la más cercana a la realidad.

Sin perjuicio de lo anterior, resultan de alto interés las diferencias observadas para grupos constituidos a partir de variables sociodemográficas, en el sentido de que el bienestar psicológico total, es diferente según estado civil y NSE. Se observa -en concordancia con la literatura- que las personas casadas muestran los promedios más altos de bienestar (Muratori y col, 2015). Igualmente interesante, resultó la inexistencia de diferencias según género lo que se opone a los estudios transculturales que son consistentes en informar diferencias de género. Los hombres tienden a mostrar mayor puntaje en autonomía y las mujeres en sus vínculos y relaciones sociales lo que no fue observado en la presente investigación (Dolan et al. 2008, Zubieta, Muratori \& Fernandez, 2012). Esta ausencia de diferencia podría explicarse porque, aunque en la cultura magallánica existe diferencia entre los roles masculinos y femeninos, en la vida familiar cotidiana ambos participan en forma similar.

Respecto de los dominios del bienestar (Laboral, Relaciones Interpersonales, Subjetivo y Material), tanto el estado civil y el NSE resultaron variables importantes. Las personas casadas evidencian mayores niveles de bienestar subjetivo mientras que las viudas y separadas presentan los menores niveles. Estas diferencias vendrían a confirmar la influencia positiva que las relaciones íntimas seguras y estables aportarían al bienestar, mientras que la disolución de las mismas resultaría perturbadora (Dolan et al. 2008). Además, la familia sigue siendo mencionada en estudios nacionales como la principal fuente de felicidad (Moyano \& Ramos, 2007) En el caso del NSE, se confirma el que disponer de recursos económicos se vincula con el bienestar subjetivo, resultado también robusto en numerosas investigaciones longitudinales que muestra, eso si, ciertas diferencias culturales (Hochman \& Skopek, 2013). 
Tanto en las dimensiones como en los dominios del bienestar, se evidenció una supremacía del estado civil "soltero" al momento de evaluar el bienestar en cuestiones de orden relacional tales como el establecimiento de vínculos sociales como el de las relaciones interpersonales. Este resultado puede deberse a que la etapa adulta joven se caracteriza por el énfasis en la búsqueda de estabilidad tanto laboral como emocional lo que se acompaña de mayor actividad social. Las personas casadas tienden a dedicar más tiempo a las redes familiares que los adultos jóvenes (Moyano \& Ramos, 2007).

Dentro de los resultados novedosos, en el dominio de las relaciones interpersonales, los participantes del NSE alto de nivel técnico, muestran mayor bienestar que los universitarios. Una posible causa de este resultado es que aquellos que pertenecen al grupo de universitarios poseen expectativas de comparación social más exigentes que aquellos del grupo de técnicos que viven su logro como un éxito (Solano \& Sánchez, 2000; Dolan et al. 2008). La diferencia entre los géneros de NSE Alto en el ámbito subjetivo, señala que en el caso de los hombres éstos presentan más bienestar que aquellos pertenecientes a otros grupos, además de que entre las mujeres esta diferencia no es significativa, lo cual podría explicarse desde el punto de vista de los estereotipo de género, ya que tales sujetos sentirían estar cumpliendo con las exigencias impuestas en relación al rol de hombre exitoso y competitivo que se espera de ellos (Bonino, 1999). Esto encuentra apoyo empírico en los resultados observados para la dimensión Felicidad.

Con relación a las múltiples dimensiones del bienestar (felicidad, autonomía, proyectos, control de situaciones, vínculos psicosociales y aceptación de si mismo), se identificaron como relevantes las variables edad, estado civil, género y NSE. Un resultado desafiante es el que indica que los adultos son aquellos que presentan mayor bienestar, cuando la literatura es consistente en indicar que los jóvenes y los adultos mayores son quienes alcanzan este nivel (Moyano \& Ramos, 2007). Una posible hipótesis seria que las condiciones climáticas y en general, las condiciones de aislamiento relativo, son vividas por las personas de estos grupos extremos de edad, como limitantes a la realización de actividades propias de su grupo etario. Los resultados similares en la dimensión "autonomía" podrían confirmar esta hipótesis. La diferencia de género observada para la dimensión "control de situaciones" que privilegia a las mujeres, podría fundamentarse también por las condiciones generales de vida en la Región que favorecen el contacto interpersonal y regulan las relaciones sociales por lo que el control percibido, sobre áreas definidas habitualmente como de interés femenino, se encuentran adecuadamente desarrolladas. A este respecto, la literatura indica que, aunque los hombres suelen presentar mayor bienestar en la dimensión "aceptación de sí mismos", lo que fue parcialmente observado en nuestra muestra (para NSE alto), las mujeres suelen mostrar mayores promedios en las dimensiones "control de situaciones" y "vínculos psicosociales" (Gonzalez, Montoya, Casullo \& Bernabéu, 2002).

Uno de los aportes de esta investigación es la de proveer información respecto a una población poco estudiada en la que la dimensión ambiental parece tener un impacto 
diferencial. La mayoría de los resultados que van en contra de la literatura existente pueden explicarse por la presencia de condiciones climáticas y de aislamiento presentes en el ambiente natural de los habitantes de la Patagonia Austral. Es sobre esta temática que resulta necesario profundizar en futuras investigaciones. Aunque el objetivo principal de este estudio fue caracterizar el bienestar subjetivo, resulta evidente que conllevan el desafío de investigar específicamente a qué se debe el impacto de las variables sociodemográficas analizadas, en el bienestar subjetivo, ya que aún no es posible determinar las fuentes de causalidad de los efectos observados. El avance en esta temática podría facilitar el desarrollo de planes de intervención en una Región geográfica con características particulares y extrapolar los resultados a otras zonas con condiciones similares.

\section{REFERENCIAS}

Arita, B. (2005). La capacidad y el bienestar subjetivo como dimensiones de estudio de la calidad de vida. Revista Colombiana de Psicología. 14, 73-79.

Ardila, R. (2003). Calidad de Vida: Una definición integradora. Revista Latinoamericana de Psicología, 35(2), 161-164.

Argyle, M. (1993). Psicología y Calidad de Vida. Madrid: Colegio Oficial de Psicólogos de Madrid.

Barrientos, J. (2006). Calidad de Vida. Bienestar Subjetivo: Una Mirada Psicosocial. Santiago: Universidad Diego Portales.

Barrio, M., \& Domenech, A. (1993). Ruptura matrimonial y salud mental: Variables psicosociales asociadas en el caso de la mujer. Revista de Psicología Social, 8(1), 33-46.

Blanchflower, D., \& Oswald, A. (2008) Is well-being U-Shaped over the life cycle? Social Sciencia \& Medicine, 66, $1733-1749$

Bonino, L. (1999). Varones, género y salud mental: desconstruyendo la normalidad masculina. En M. Segarra \& A. Carabí (Eds.), Nuevas masculinidades. Barcelona: Icaria.

Borthwick-Duffy, S. A. (1992). Quality of life and quality of care in mental retardation. In L. Rowitz (Ed.), Mental Retardation in the Year 2000. Disorders of Human Learning, Behavior, and Communication (pp. 52-66). New York: Springer.

Carr, A. (2007). Psicología Positiva. La ciencia de la felicidad. Ed. Paidós.

Castro Solano, A. (2002). Investigaciones argentinas sobre el bienestar psicológico, en M. Casullo (Comp.), Evaluación del bienestar psicológico en Iberoamérica. Buenos Aires: Paidós.

Casullo, M., \& Brenlla, M. (2002). Evaluación del Bienestar Psicológico en Iberoamérica. Buenos Aires: Paidós.

Contini, N., Cohen, S., Figueroa, M., \& Coronel, P. (2006) Pensar la Adolescencia hoy: De la psicopatología al bienestar psicológico. Buenos Aires: Paidós.

Cuadra, H., \& Florenzano, R. (2003). Bienestar Subjetivo: Hacia una psicología positiva. Revista 
de Psicología de la Universidad de Chile, 12(1), 86-96.

Díaz, D., Rodríguez, R., Blanco, A., Moreno, B., Gallardo, I., Valle, C., \& Dierendonck, D. (2006). Adaptación española de las escalas de bienestar psicológico de Ryff. Psicothema, 18(3), 572-577.

Diener, E., \& Biswas, R., (2002) Will Money Increase Subjective Well-Being? A literature review and Guide to Needed Research. Social Indicators Research, 57(2), 119-169.

Dolan, P., Peasgood, T., \& White, M. (2008) Do we really know what makes us happy? Review of the economic literature on the factors asociated with subjective well-being. Journal of Economic Psychology, 29, 94-122.

Estrada, C., Cirilo, S., Torres, E., \& Valderrama, S. (2005). Manual Operativo de Metodología Cuantitativa. FONAD 2005, UMAG

Garduño, L., Salinas, B., \& Rojas, M. (2005) Calidad de Vida y Bienestar Subjetivo en México. México, D.F.: Plaza y Valdés.

Gómez, M., \& Sabeh, E. (2001). Calidad de vida: Evolución del concepto y su influencia en la investigación y la práctica. Salamanca: Instituto Universitario de Integración en la Comunidad, Facultad de Psicología, Universidad de Salamanca.

González, R., Montoya, I., Casullo, M., \& Bernabéu, J. (2002) Relación entre estilos y estrategias de afrontamiento y bienestar psicológico en adolescentes. Psicothema, 14(2), 363-368.

Hochman, O., \& Skopek, N. (2013). The impact of wealth on subjective well-being: A comparaison of three welfare-state regimes. Research in Social Stratification and Mobility, 34(1), 127141.

Keyes, C., Shmotkin, D., \& Ryff, C. (2002). Optimizing Well-Being: The Empirical Encounter of Two Traditions. Journal of Personality and Social Psychology, 82(6), 1007-1022.

Moyano, E., \& Ramos, N. (2007) Bienestar subjetivo: midiendo satisfaccion vital, felicidad y salud en poblacion chilena de la región del Maule. Universum, 22(2), 184-200.

Muratori, M., Zubieta, E., Ubillos, S., Gonzáles, J. L., \& Bobowik, M. (2015). Felicidad y Bienestar Psicológico: Estudio Comparativo Entre Argentina y España. Psykhe 24(2), 1-18

Organización Mundial de la Salud (2001). Informe sobre la salud en el mundo. Salud mental: nuevos conocimientos, nuevas esperanzas.

PNUD (1990). Informe sobre el desarrollo humano: Definición y Medición del Desarrollo Humano.

Sánchez-Cánovas, J. (1998). Escala de Bienestar Psicológico. Buenos Aires: TEA Ediciones.

Suh, E., Diener, E., \& Fujita, F. (1996) Events and Subjective Weel-Being: Only Recent Events Matter. Journal of Personality and Social Psychology, 70(5), 1091-1102.

Ryan, R., \& Deci, E. (2001). On Hapiness and Human Potentials: A Review of Research on Hedonic and Eudaimonic Well-Being. Annual Review Psychology, 52, 141-166.

Veenhoven, R. (2000). The four qualities of life. Ordering concepts and measures of the good life. Journal of happiness studies, 1(1), 1- 39.

Wildman, J., \& Jones, A. (2002). Is it absolute income or relative deprivation that leads to poor 
psychological well being? A test based on individual-level longitudinal data. University of York: YSHE

Whoqol (1995). The World Health Organization Quality of Life assessment (WHOQOL): position paper from the World Health Organization. Social Science \& Medicine, 41(10),1403-9

Zubieta, M., Muratori, M., \& Fernandez, O. (2012). Bienestar subjetivo y psicosocial: explorando diferencias de género. Salud \& Sociedad, 3(1), 66-76. 